\title{
Spiral QAM Modulated CO-OFDM System with Increased Tolerance toward Laser Phase Noise
}

\author{
Chong Li \\ College of Information Science and Engineering \\ Hunan University \\ Changsha, China \\ e-mail: 814616418@qq.com
}

\author{
Lin Chen \\ College of Information Science and Engineering \\ Hunan University \\ Changsha, China \\ e-mail: liliuchen12@vip.163.com
}

\begin{abstract}
To mitigate the phase noise, we propose Spiral QAM in CO-OFDM. Simulation results, based on 40-GS/s COOFDM systems, show that 16 Spiral QAM can increase the phase noise tolerance, as compared to square 16 QAM.

Keywords-Coherent optical orthogonal frequency-division Multiplexing (CO-OFDM), phase noise, 16 Spiral QAM.
\end{abstract}

\section{INTRODUCTION}

Coherent optical orthogonal frequency-division multiplexing (CO-OFDM) is a promising modulation scheme for high speed optical communications [1]. COOFDM brings to optical communication, which is the combination of two powerful techniques, coherent optical detection and OFDM technique [2]. It has been recently proposed to combat fiber chromatic dispersion and polarization-mode dispersion (PMD) [3, 4]. To make use of the available spectrum in optical fibers efficiently, square 16 QAM constellations making a good compromise between spectral efficiency and reach is adopted widely [5]. However, OFDM is prone to phase noise, which will cause both common phase error (CPE) and inter-carrier interference (ICI) [6]. Moreover, square 16 QAM constellations is optimum only for additive white Gaussian noise (AWGN) channels. Phase noise originating from lasers cannot be neglected in optical long-haul links [7]. To realize phase noise suppression, several methods have been proposed, in which CPE is identified and ICI is approximated as an additive Gaussian noise [8,9]. However, only suppressing $\mathrm{CPE}$ is not sufficient for relatively larger laser line width. Moreover, the cost of the CO-OFDM system will be increased as the laser line width become narrower. Estimation and compensation of both CPE and partial ICI in CO-OFDM system were proposed [10, 11]. These schemes use iterative algorithms for joint estimate the data and the phase noise vector. The common drawback of iterative algorithms is that they suffer from large latency and high implementation complexity, which is unsuitable for future real-time CO-OFDM systems. Although those methods can improve the performance of the CO-OFDM system with the influence on the phase noise, there is no consideration about adopting a new 4 bit/symbol constellations to restrain the phase noise in CO-OFDM.

The Spiral QAM was initially proposed in wireless single carrier communication systems [12]. However, the analysis, focused on phase noise of Gaussian distribution, is not enough for CO-OFDM system with laser noise modeled as Wiener process [13]. In this letter, 16 Spiral QAM modulation format that considers both AWGN and phase noise is introduced into CO-OFDM system for the first time. A detailed comparison between 16 Spiral QAM and square 16 QAM is presented. By means of theory and numerical simulation, it is shown that 16 Spiral QAM for the COOFDM system can improve the tolerance to the phase noise effectively. Moreover, the power efficiency and other features of 16 Spiral QAM is close to conversional square 16 QAM.

\section{SPIRAL QAM MODULATED CO-OFDM SYSTEM}

\section{A. Setup of CO-OFDM Systems}

Fig. 1 shows the block diagram of 40GS/s coherent optical OFDM system. The OFDM signal is composed by 256 subcarriers from which 192 subcarriers carry data information, 8 are pilot subcarriers used for the maximumlikelihood (ML) phase estimation [9] and 56 subcarriers are guard subcarriers. The length of cyclic prefix for every OFDM symbol is 32 . There are 2 training sequence in front of OFDM signal. The first one is used for the frame synchronization [14], and the other is inserted for channel estimation. Because energy loss will increase with longer cyclic prefix and the dispersion impulse response is of finite duration, the finite impulse response (FIR) filter can be used for dispersion compensation [15]. The transmission of signal is composed by 2 training sequence and 48 OFDM signals. The sampling rate of DAC is 40GS/s. In the optical link, the launch power of lasers is assumed to be $0 \mathrm{dBm}$. Then a recirculating loop is considered for transmission purposes. The loop has an $80 \mathrm{~km}$ standard single mode fiber (SSMF) with a dispersion parameter of $16 \mathrm{ps} / \mathrm{nm} / \mathrm{km}$ and a gain controlled EDFA. The variable OSNR block(in a reference optical bandwidth of $0.1 \mathrm{~nm}$ ) consist of a variable optical attenuator following the EDFA with $4 \mathrm{~dB}$ of noise figure, which was used to control the signal power entering the optic-electronic receiver frontend[16]. At the receiver, the coherent detector consists of a local oscillator (LO), a $90^{\circ}$ 
hybrid, and two balanced detectors. Then the last electrical signal processed by MATLAB.
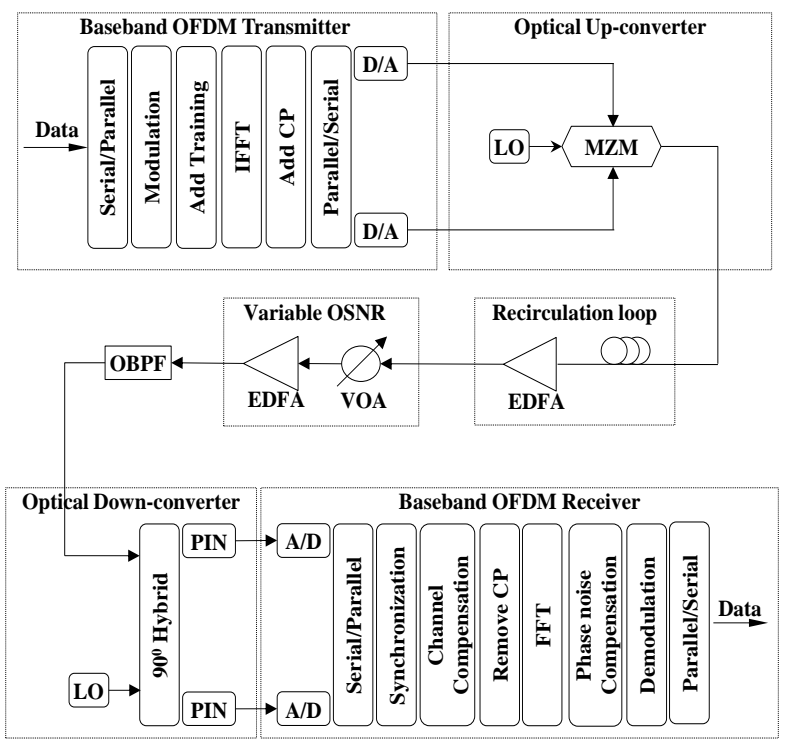

Figure 1. Block diagram of the CO-OFDM system setup.

\section{B. 16 Spiral QAM}

In 16 QAM modulations, one symbol represents four bits. We can map the binary sequence as the corresponding 16 QAM constellation as long as the mapping rule is known. Based on [12], the constellation of 16 Spiral QAM can be obtained. Usually, gray mapping is used in combination with the conventional 16 QAM [17], which the first two bits are used for the generation of in-phase component (I) and the last two are used for the generation of quadrature component (Q), the generated symbol is $I+j Q$. However, gray mapping is unavailable for the unique constellation structure of Spiral QAM. The appropriate symbol mapping rule for 16 Spiral QAM is shown in Fig.2. In order to understand the advantage of Spiral QAM, we concentrate on analyzing the structural features of 16 Spiral QAM.

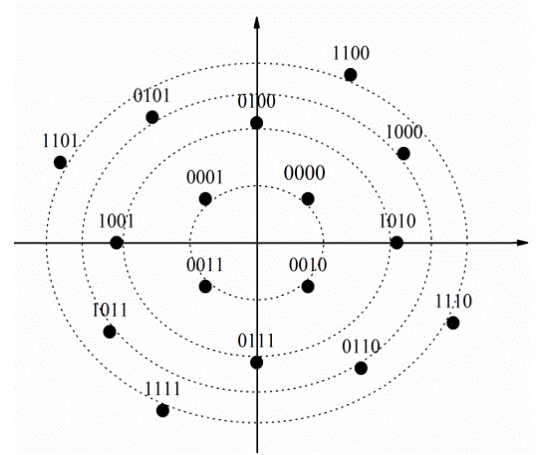

Figure 2. Symbol mapping for 16 Spiral QAM.

In the outskirt of the IQ-plane, the next symbol locations of Spiral QAM are determined by not only the minimum angular distance but also the minimum Euclidean distance between adjacent symbols. In order to resist the phase noise, 16 Spiral QAM sets a lager minimum angular distance than square 16 QAM. The performance of the constellations is evaluated by the method using the constellation points [18]. Assuming that the average power of Gaussian noise is $2 N_{0}$ and all the signals in the set share the equal probability of transmitting. The theoretical average symbol error rate based on constellation in the presence of phase noise can be expressed as [18]

$$
\left\{\begin{array}{c}
P e \approx \frac{1}{N} \sum_{T} \sum_{R \neq T} \frac{1}{A \sqrt{2 \pi}} e^{-\frac{A^{2}}{2}} \\
A=\frac{\left(S_{R}-S_{T}\right)\left[S_{R}+S_{T}\left(1-2 e^{j \varphi}\right)\right]}{2 \sqrt{N_{0}}\left\|S_{R}-S_{T}\right\|}
\end{array}\right.
$$

Where $S_{R}$ and $S_{T}$ are the transmitted and the received signal, respectively. $R$ and $T$ represent the constellation of the corresponding transmitted and the received signal, respectively. $N$ is the total number of the constellation points. $A$ is a variable to simplify the expression. " $\bullet$ " represents dot product. "I||" represents the amplitude of a complex number. $\varphi$ is the phase noise.

And in order to calculate SNR, we need normalized the average power of the constellation. The new constellation points $C_{n}$ is given by

$$
C_{n}=\sqrt{S_{n}} \sqrt{\frac{\sum_{k=1}^{N}\left\|S_{k}\right\|^{2}}{N}} \quad k=1,2,3, \ldots N
$$

Here $S_{n}$ are the original constellation points.

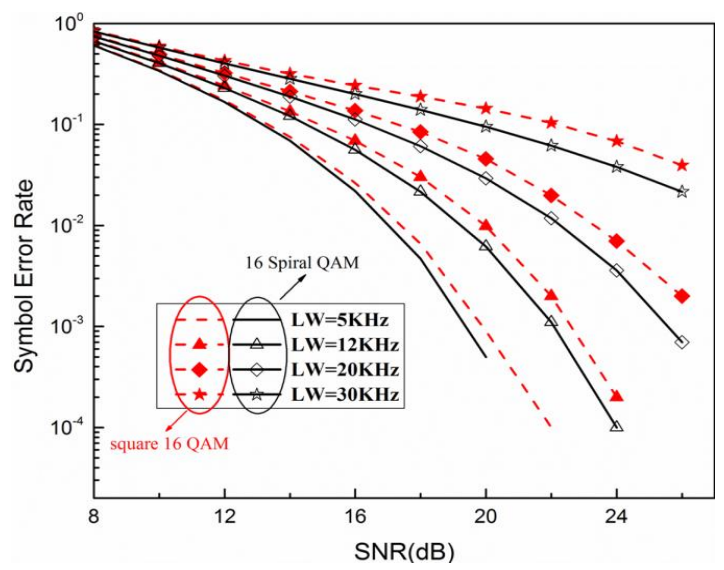

Figure 3. SER curves of 16 Spiral QAM and the conventional 16 QAM for various values of phase error modeled as Wiener process in theory.

We can get the relationship between symbol error rate and SNR in the presence of phase noise through (1), which is shown in Fig. 3. Furthermore, from theoretical results depicted in Fig. 3, we notice that 16 Spiral QAM achieve a 
performance similar to square 16 QAM as the SNR increased. To achieve $S E R=10^{-3}$, square 16 QAM requires a $0.6 \mathrm{~dB}$ higher SNR when line width is $12 \mathrm{kHz}$. And the superiority of 16 Spiral QAM will be more obvious while the line width become larger. The detail analysis will be drawn in the next Section.

\section{SIMULATION RESULTS AND ANALYSIS}

We first consider a back-to-back system with a laser of various line widths, which two different modulated signals are used in the transmitter. We assume that the line width of transmitter and LO lasers are the same.

The performance of Symbol Error Ratio (SER) against the OSNR of received square 16 QAM and 16 Spiral QAM modulated OFDM signal is simulated for laser line width of $5 \mathrm{kHz}, 12 \mathrm{kHz}, 20 \mathrm{kHz}$ and $30 \mathrm{kHz}$, respectively, as shown in Fig. 4.

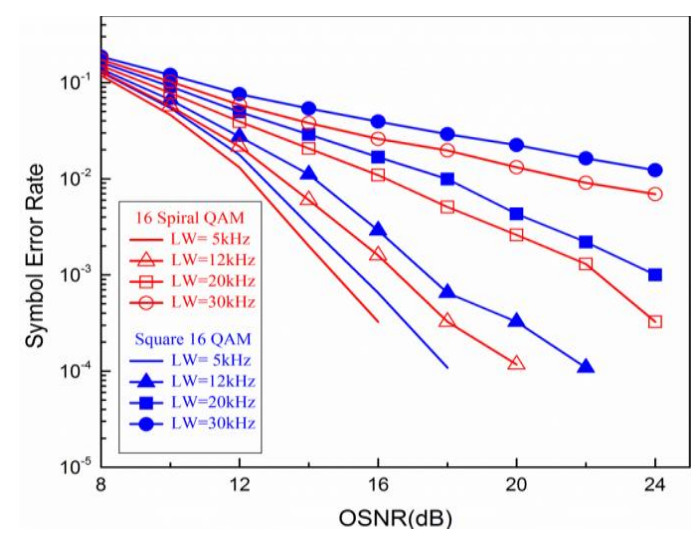

Figure 4. SER versus OSNR for various line widths in back-to-back case

As in term of line width, the results are the same with the theoretical results as shown in Fig. 3. Because of the same Euclidean distance between each constellation point, two different QAM modulations show comparable performance with the narrow line width. As the line width became lager, the performance of the square 16 QAM degrades more considerably than 16 Spiral QAM. To achieve $S E R=10^{-3}$ with $12 \mathrm{kHz}$ line width, the required OSNR value for 16 Spiral QAM modulated OFDM is about $16.7 \mathrm{~dB}$, and for square 16 QAM modulated OFDM is $17.5 \mathrm{~dB}$. When the line width is $20 \mathrm{kHz}$, the required OSNR for a SER of $10^{-3}$ is $22.3 \mathrm{~dB}$ and $24 \mathrm{~dB}$ for 16 Spiral QAM and square 16 QAM, respectively. Therefore, the OSNR improvement is increased to $1.7 \mathrm{~dB}$. To achieve $S E R=10^{-2}$, square $16 \mathrm{QAM}$ requires about a $2 \mathrm{~dB}$ higher OSNR than 16 Spiral QAM when the line width increased to $30 \mathrm{kHz}$. This is due to the fact that 16 Spiral QAM possesses 4 amplitude rings, each carrying 4 points with an $90^{\circ}$ angular distance between two adjacent points in the same radius, while square 16 QAM is made up of 3 amplitude rings and the minimum angular distance is $37^{\circ}$ in the middle ring. The angular distance determines the performance of the constellation against the phase noise.

Then the phase noise tolerance was considered in case of back-to-back and after $800 \mathrm{~km}$ transmission. The maximum- likelihood (ML) phase estimation [9] is used to mitigate partial phase noise with the laser line width lager than 200 $\mathrm{kHz}$. Considering the advantage on phase noise, no matter which case 16 Spiral QAM will perform better. As depicted in the Fig. 5, the proposed 16 QAM clearly outperforms square 16 QAM over the whole studied laser phase noise line width region from 200 to $2000 \mathrm{kHz}$ with a fixed OSNR of $22 \mathrm{~dB}$. Because ML phase estimation works effectively to the laser with a line width less than $1 \mathrm{MHz}$ [9], the performance of 16 Spiral QAM is near to square 16 QAM with line width less than $1 \mathrm{MHz}$. Furthermore, 16 Spiral QAM is still useful to residual phase noise after ML phase estimation with lager laser line width.

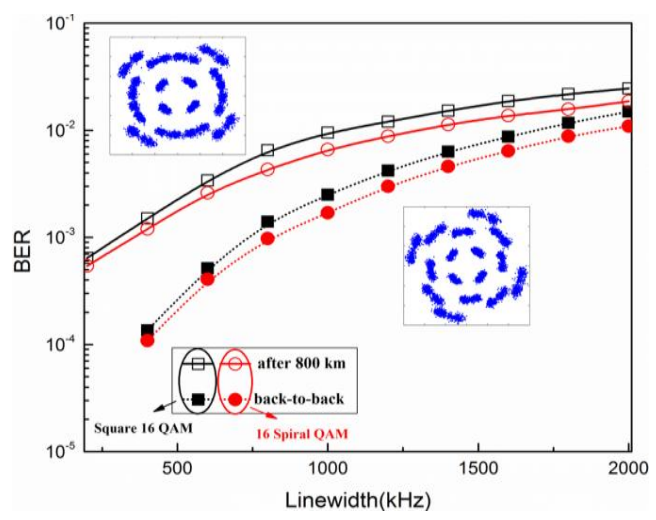

Figure 5. Simulated BER as a function of line width with fixed received OSNR of $22 \mathrm{~dB}$ in case of back-to-back and after $800 \mathrm{~km}$ transmission.

As the ASE noise decrease OSNR of the system, the tolerance towards line width becomes lower after $800 \mathrm{~km}$ transmission, comparing the back-to-back case. However, 16 Spiral QAM still improve noise tolerance of CO-OFDM than square 16 QAM after $800 \mathrm{~km}$ transmission, as well as in back-to-back case. For the CO-OFDM system of $800-\mathrm{km}$ transmission, a benefit of about $100 \mathrm{kHz}$ is possible for the maximum line width. The close Euclidean distance of two QAM modulation scheme guarantee that their performance against the additive noise is close. Moreover, 16 Spiral QAM make full use of the same power with square 16 QAM by the rational constellations arrangement. Obviously, 16 Spiral QAM exceeds square 16 QAM in the presence of phase noise without degrading the performance towards ASE noise tolerance, namely the advantage on phase noise of 16 Spiral QAM is very stable compared to square 16 QAM.

\section{SUMMARY}

In this paper, we have proposed 16 Spiral QAM encoded coherent optical OFDM for the first time. Although the laser phase noise is a stochastic Wiener processes unlike the Gaussian noise, the constellation of 16 Spiral QAM is still optimized. Due to the angular distance between two adjacent points of 16 Spiral QAM is larger than Square 16 QAM, and the performance of two different QAM is discussed theoretically, the phase noise tolerance of 16 Spiral QAM with CO-OFDM is enhanced. After mixed in ASE noise through the fiber transmission, the advantage on 
phase noise of 16 Spiral QAM is similar to back-to-back case. In addition, some methods such as the minimum distance detection, dispersion compensation and maximumlikelihood phase estimation can be used for 16 Spiral QAM like the conventional 16 QAM.

\section{ACKNOWLEDGMENT}

This work is supported by the National Natural Science Foundation of China $(61307087,61377079)$, by Hunan Provincial Natural Science Foundation of China (12JJ3070), by the National "863" High Tech Research and Development Program of China (2011AA010203), and by the Fundamental Research Funds for the Central Universities and Young Teachers Program of Hunan University.

\section{REFERENCES}

[1] J. Armstrong, "OFDM for Optical Communications." Lightwave Technology, Journal of. 27(03), 189-204 (2009).

[2] Liu X, Liang H, Dai B, et al. "Mth-power-law method to compensate laser linewidth of $100 \mathrm{~Gb} / \mathrm{s}$ PDM-CO-OFDM systems." Chin. Opt. Lett., Journal of. 10(s1): 10609(2012).

[3] W. Chung, "A Matched Filtering Approach for Phase Noise Suppression in CO-OFDM Systems." Photonics Technology Letters, IEEE. 22(24), 1802-1804(2010).

[4] Wu, Yiling, et al. "Coherent optical OFDM scheme with inter-carrier interference self-cancellation and common phase error compensation." Chin. Opt. Lett., Journal of. 08(07), 634-638-5 (2010).

[5] R. Essiambre, G. Kramer, P. J. Winzer, G. J. Foschini, and B. Goebel, "Capacity Limits of Optical Fiber Networks." Lightwave Technology, Journal of. 28(04), 662-701(2010).

[6] V. Syrjala and M. Valkama, "Receiver DSP for OFDM Systems Impaired by Transmitter and Receiver Phase Noise," in Communications (ICC), 2011 IEEE International Conference on, pp. 1-6(2011).

[7] T. Pfau, X. Liu, and S. Chandrasekhar, "Optimization of 16-ary Quadrature Amplitude Modulation constellations for phase noise impaired channels." in European Conference and Exposition on Optical Communications, (2011).
[8] Y. Xingwen, W. Shieh, and T. Yan, "Phase Estimation for Coherent Optical OFDM," Photonics Technology Letters, IEEE. 19(12), 919921(2007).

[9] W. Shieh, "Maximum-Likelihood Phase and Channel Estimation for Coherent Optical OFDM." Photonics Technology Letters, IEEE. 20(08), 605-607(2008).

[10] Y. Chuanchuan, Y. Feng, and W. Ziyu, "Orthogonal Basis Expansion-Based Phase Noise Estimation and Suppression for COOFDM Systems." Photonics Technology Letters, IEEE. 22(01), 5153(2010).

[11] Y. Chuanchuan, H. Changhong, and W. Ziyu, "Iterative phase noise estimation and suppression for CO-OFDM systems with large laser linewidth." in Communications and Photonics Conference and Exhibition, 2011. ACP. Asia, 1-6(2011).

[12] K. Byung-Jae, S. Nah-Oak, P. Bumsoo, and K. Dong Seung, "Spiral QAM: A Novel Modulation Scheme Robust in the Presence of Phase Noise." in Vehicular Technology Conference, 2008. VTC 2008-Fall. IEEE 68th, 1-5(2008).

[13] D. Petrovic, W. Rave, and G. Fettweis, "Effects of Phase Noise on OFDM Systems With and Without PLL: Characterization and Compensation." Communications, IEEE Transactions on. $\mathbf{5 5}(08)$, 1607-1616(2007).

[14] Minn, Hlaing, Vijay K. Bhargava, and Khaled Ben Letaief. "A robust timing and frequency synchronization for OFDM systems." Wireless Communications, IEEE Transactions on 02(04), 822-839(2003).

[15] Savory S J. "Digital filters for coherent optical receivers," Optics Express, 16(02), 804-817 (2008).

[16] Cartaxo, Adolfo VT, and Tiago MF Alves, "Theoretical and experimental performance evaluation methods for DD-OFDM systems with optical amplification." Journal of Microwaves, Optoelectronics and Electromagnetic Applications 10(01), 82-94 (2011).

[17] W. Hui, L. Yan, Y. Xiaogang, K. Deming, W. Jian, and L. Jintong, "APSK Modulated CO-OFDM System With Increased Tolerance Toward Fiber Nonlinearity." Photonics Technology Letters, IEEE. 24(13), 1085-1087(2012).

[18] L. Yang, X. Shuzheng, and Y. Huazhong, "Design of Circular Signal Constellations in the Presence of Phase Noise." in Wireless Communications, Networking and Mobile Computing, 2008. WiCOM '08. 4th International Conference on, 1-8(2008). 